\title{
Molecular studies of Planetary Nebulae
}

\author{
Yong Zhang ${ }^{1,2}$ \\ ${ }^{1}$ Department of Physics, The University of Hong Kong, Pokfulam Road, Hong Kong, China \\ ${ }^{2}$ Laboratory for Space Research, Faculty of Science, The University of Hong Kong, Pokfulam \\ Road, Hong Kong, China \\ email: zhangy96@hku.hk
}

\begin{abstract}
Circumstellar envelopes (CEs) around evolved stars are an active site for the production of molecules. After evolving through the Asymptotic Giant Branch (AGB), proto-planetary nebula $(\mathrm{PPN})$, to planetary nebula $(\mathrm{PN})$ phases, CEs ultimately merge with the interstellar medium (ISM). The study of molecules in PNe, therefore, is essential to understanding the transition from stellar to interstellar materials. So far, over 20 molecular species have been discovered in PNe. The molecular composition of PNe is rather different from those of AGB and $\mathrm{PPNe}$, suggesting that the molecules synthesized in PN progenitors have been heavily processed by strong ultraviolet radiation from the central star. Intriguingly, fullerenes and complex organic compounds having aromatic and aliphatic structures can be rapidly formed and largely survive during the PPN/PN evolution. The similar molecular compositions in $\mathrm{PNe}$ and diffuse clouds as well as the detection of $\mathrm{C}_{60}^{+}$in the ISM reinforce the view that the mass-loss from PNe can significantly enrich the ISM with molecular species, some of which may be responsible for the diffuse interstellar bands. In this contribution, I briefly summarize some recent observations of molecules in $\mathrm{PNe}$, with emphasis on their implications on circumstellar chemistry.
\end{abstract}

Keywords. planetary nebulae, circumstellar matter, astrochemistry, molecular processes, ISM: molecules, infrared: ISM

\section{Introduction}

The mass loss of evolved stars with intermediate-mass progenitors creates an expanding circumstellar envelope $(\mathrm{CE})$, in which a variety of molecules are synthesized. During the CE evolution from the Asymptotic Giant Branch (AGB), proto-planetary nebula (PPN), to planetary nebula $(\mathrm{PN})$ phases, the physical conditions dramatically alter, resulting in considerable changes in molecular composition. The yielded molecules eventually disperse into the interstellar medium (ISM), and may act as the seed of complex organic molecules discovered in molecular clouds (Ziurys 2008). Therefore, the investigation of molecules in $\mathrm{PNe}$ provides essential information on the material cycle in galaxies. The very existence of molecular gas in PNe can be inferred from the large discrepancy between the mass of the PN progenitor $\left(1-8 M_{\odot}\right)$ and the masses of PN central stars and ionized nebulae $\left(\sim 0.9 M_{\odot}\right)$, known as the missing mass problem (Kwok 1994).

Up to now more than 80 different molecular species have been detected in C-rich AGB envelopes and PPNe. Numerous models have been developed to simulate the chemical processes in AGB envelopes (see Millar 2016, for a recent review). The standard picture is that the parent molecules are formed through equilibrium chemistry near the stellar photosphere, and then accelerated by radiation pressure on dust into outer regions where more complex molecules are synthesized by the chemistry induced by the external interstellar ultraviolet radiation field. The chemical evolution during the AGB-PPN-PN transitions has been investigated through systematic molecular line surveys (see Bujarrabal 2006, Cernicharo et al. 2011, for detailed reviews), which clearly imply dramatic changes of molecular composition at the PPN phase (e.g. Pardo et al. 2007, Park et al. 
Table 1. The molecules detected in PNe.

\begin{tabular}{lllll}
\hline $\begin{array}{l}\mathbf{2} \\
\text { atoms }\end{array}$ & $\begin{array}{l}\mathbf{3} \\
\text { atoms }\end{array}$ & $\begin{array}{l}\mathbf{4} \\
\text { atoms }\end{array}$ & $\begin{array}{l}\mathbf{5} \\
\text { atoms }\end{array}$ & $\begin{array}{l}>5 \\
\text { atoms }\end{array}$ \\
\hline $\mathrm{H}_{2}$ & $\mathrm{C}_{2} \mathrm{H}$ & $\mathrm{NH}_{3}$ & $c-\mathrm{C}_{3} \mathrm{H}_{2}$ & $\mathrm{C}_{60}$ \\
$\mathrm{CH}$ & $\mathrm{HCN}$ & $\mathrm{H}_{2} \mathrm{CO}$ & $\mathrm{HC}_{3} \mathrm{~N}$ & $\mathrm{C}_{60}^{+}$ \\
$\mathrm{CH}^{+}$ & $\mathrm{HCO}^{+}$ & & & $\mathrm{C}_{70}$ \\
$\mathrm{CN}$ & $\mathrm{H}_{2} \mathrm{O}$ & & & \\
$\mathrm{CO}$ & $\mathrm{HNC}^{+}$ & & & \\
$\mathrm{CO}^{+}$ & $\mathrm{N}_{2} \mathrm{H}^{+}$ & & & \\
$\mathrm{OH}^{+}$ & $\mathrm{SO}_{2}$ & & & \\
$\mathrm{OH}^{+}$ & $\mathrm{HCS}^{+}$? & & & \\
$\mathrm{SiO}$ & $\mathrm{SiC}_{2}$ ? & & & \\
$\mathrm{CS}$ & & & & \\
$\mathrm{SO}$ & & & & \\
$\mathrm{NS}$ & & & & \\
\hline
\end{tabular}

Note: Not including the molecular-rich pre-PN CRL 618 and solid-phase species.

2008, Zhang et al. 2013). The dynamical timescales of the three evolutionary stages can impose constraints on the efficiencies of chemical reactions. Thus CEs of evolved stars can serve as a laboratory for studying the chemical processes under extreme conditions.

With the evolution to the PN phase, increasing central-star temperature results in a photoionized region surrounded by a photon-dominated region (PDR), where the chemistry is dominated by the photons of energy from 6 to $13.6 \mathrm{eV}$, which contribute significantly to gas heating by ejecting electrons from dust grains and induce ion-neutral reactions. Shocks may play an important role in circumstellar chemistry by inducing endothermic reactions and destructing dust into molecules, though strong shocks might destroy molecules. Although high photodissociation and dissociative recombination rates render the environments of $\mathrm{PNe}$ hostile for the survival of molecules, the ultraviolet radiation can be largely attenuated by clumps, density-enhanced tori, as well as self- and mutual-shielding of molecules. These optically thick regions and the extensive gas envelopes presumably have different chemical evolution routes from one another. The first chemical model of PNe was developed by Black (1978) who predicted the existence of simple molecules such as $\mathrm{H}_{2}, \mathrm{H}_{2}^{+}, \mathrm{HeH}^{+}, \mathrm{OH}$, and $\mathrm{CH}^{+}$in PDRs. Although most of them were confirmed in subsequent observations, $\mathrm{HeH}^{+}$, a molecule of cosmological interest, remains undetected. To date, various models, including equilibrium and time-dependent models, have been constructed to study the chemistry in envelopes and clumps, but none of them is fully satisfying (Kimura 2012, and reference therein).

Early observational studies of molecules in PNe mainly focused on $\mathrm{H}_{2}$ and $\mathrm{CO}$ (e.g. Zuckerman \& Gatley 1988, Huggins et al. 1996). Later on, millimetre observations were undertaken to trace the chemical variations of PNe (Bachiller et al. 1997, Josselin \& Bachiller 2003), which indicated an appreciable enrichment of $\mathrm{HCO}^{+}$and $\mathrm{CN}$ radicals in PNe with respect to AGB envelopes. Infrared observations have proven to be useful for searching for new small molecules (Cernicharo et al. 1997, Liu et al. 1997) and fullerenes (Cami et al. 2010, García-Hernández et al. 2010) in PNe. The list of molecules in PNe continues to lengthen with more sensitive radio observations (e.g. Zhang et al. 2008, Tenenbaum et al. 2009, Edwards \& Ziurys 2013,2014, Schmidt \& Ziurys 2016) and the launch of the Herschel Space Observatory (e.g. Bujarrabal et al. 2012, van de Steene, this volume). Table 1 shows the molecules discovered so far in PNe. Although most of them are simple molecules, a big gap exists between the molecules with 5 and 60 atoms. It is reasonable to hypothesize that complex molecules ( $\geqslant 6$ atoms) are generally present in $\mathrm{PNe}$, and it is worthwhile to pursue future observations to search for the missing 
molecules. The aim of this paper is to selectively review the studies of molecules observed in $\mathrm{PNe}$, mostly focusing on the results published in the last few years.

\section{Small gas-phase molecules}

$\mathrm{H}_{2}$ and $\mathrm{CO}$. The number of $\mathrm{PNe}$ harboring $\mathrm{H}_{2}$ has recently been considerably increased by the UWISH2 survey (Froebrich et al. 2015, Gledhill, this volume), in which 284 extended $\mathrm{H}_{2}$ sources are identified as PNe or PN candidates. $\mathrm{H}_{2}$ emission appears stronger in bipolar PNe with equatorial rings (Marquez-Lugo et al. 2013, and reference therein). Manchado et al. (2015 and this volume) presented high-resolution $\mathrm{H}_{2}$ images showing that the $\mathrm{H}_{2}$ emission in the central torus appears clumpy rather than uniform, and suggested that those clumps might eventually populate the ISM and contribute to the baryonic dark matter. Based on the Herschel observations of an evolved PN NGC 6720, van Hoof et al. (2010) concluded that $\mathrm{H}_{2}$ could be reformed on dust grains after the central star enters the cooling track. So far more than 80 PNe have been detected with CO (Huggins et al. 1996, 2005). Early observations revealed large variations in the CO properties from object to object, with young PNe exhibiting larger $\mathrm{CO}$ mass than evolved ones (Bujarrabal 2006). However, recent observations show that the CO abundance does not significantly vary during PN evolution (Edwards et al. 2014), suggesting that substantial amount of molecules can be expelled from PNe into the ISM.

$\mathrm{H}_{2} \mathrm{O}$ and $\mathrm{OH}$. When $\mathrm{CEs}$ enter the $\mathrm{PN}$ phase, $\mathrm{H}_{2} \mathrm{O}$ and $\mathrm{OH}$ masers will rapidly disappear $\left(<10^{3}\right.$ yrs $)$, and thus can trace young $\mathrm{PNe}$. To date, five $\mathrm{PNe}$ with $\mathrm{H}_{2} \mathrm{O}$ and seven with $\mathrm{OH}$ maser emission have been confirmed, among which only two exhibit both maser emission (Gomez et al. 2015a, Uscanga et al. 2012, Qiao et al. 2016). Gomez et al. (2015b) discovered the first "water fountain" PN that was speculated to be the youngest $\mathrm{PN}$ known so far. $\mathrm{H}_{2} \mathrm{O}$ and $\mathrm{OH}$ infrared emission has been detected in the O-rich PN NGC 6302 (Bujarrabal et al. 2012) and the C-rich PN NGC 7027 (Liu et al. 1996, Wesson et al. 2010). A possible explanation for the presence of oxygen compounds in C-rich environments is that the $\mathrm{PN}$ might have experienced a transition from $\mathrm{O}$ - to C-rich chemistry following a helium shell flash. However, a more plausible explanation is that they origin from an ongoing photo-induced process in PDRs (Santander-García et al. 2012).

$\mathrm{OH}^{+}, \mathrm{CO}^{+}$, and $\mathrm{HCO}^{+}$. The three cationic molecules can be formed in PDRs through the path $\mathrm{CO} \stackrel{h v}{\longrightarrow} \mathrm{O} \stackrel{\mathrm{H}^{+}}{\longrightarrow} \mathrm{O}^{+} \stackrel{\mathrm{H}_{2}}{\longrightarrow} \mathrm{OH}^{+} \stackrel{\mathrm{H}_{2}}{\longrightarrow} \mathrm{H}_{2} \mathrm{O}^{+} \stackrel{\mathrm{H}_{2}}{\longrightarrow} \mathrm{H}_{3} \mathrm{O}^{+} \stackrel{\mathrm{e}}{\rightarrow} \mathrm{OH} \stackrel{\mathrm{C}^{+}}{\longrightarrow} \mathrm{CO}^{+} \stackrel{\mathrm{H}_{2}}{\longrightarrow}$ $\mathrm{HCO}^{+}$. For the first time, $\mathrm{OH}^{+}$was discovered in both young and evolved $\mathrm{PNe}$ by the Herschel Space Observatory (Aleman et al. 2014, Etxaluze et al. 2014). In evolved PNe, direct photoionization of $\mathrm{CO}$ provides an alternative route to produce $\mathrm{CO}^{+}$. Bell et al . (2007) detected $\mathrm{CO}^{+}$in a sample of $\mathrm{PNe}$ with $\mathrm{HCO}^{+}$emission. The correlation between the $\mathrm{CO}^{+}$and $\mathrm{HCO}^{+}$line intensities provides support for the formation route of $\mathrm{HCO}^{+}$ from $\mathrm{CO}^{+} . \mathrm{HCO}^{+}$is a commonly detected molecule in PNe. Bachiller et al. (1997) found that $\mathrm{HCO}^{+}$abundance increases by two orders of magnitude during the PPN-PN transition. Recent observations suggest that the $\mathrm{HCO}^{+}$abundance is not closely related to the ages of PNe (Fig. 1). In highly $\mathrm{HCO}^{+}$or $\mathrm{OH}^{+}$enriched PNe, soft X-ray from the center may play an important role in chemistry, which results in abundant $\mathrm{H}_{3}^{+}$and then enhances the reaction $\mathrm{CO} \stackrel{\mathrm{H}_{3}^{+}}{\longrightarrow} \mathrm{HCO}^{+}$or $\mathrm{O} \stackrel{\mathrm{H}_{3}^{+}}{\longrightarrow} \mathrm{OH}^{+}$.

$\underline{\mathrm{CH} \text { and } \mathrm{CH}^{+}}$. Methylidyne radical and its cation, the important parent species leading to the formation of $\mathrm{CN}$ and $\mathrm{HCN}$, have been detected in the infrared spectrum of NGC 7027 (Cernicharo et al. 1997, Liu et al. 1997, Wesson et al. 2010). They can be formed through the endothermic reaction $\mathrm{C}^{+}+0.4 \mathrm{eV} \stackrel{\mathrm{H}_{2}}{\longrightarrow} \mathrm{CH}^{+}$followed by $\mathrm{CH}^{+} \stackrel{\mathrm{H}_{2}}{\longrightarrow}$ 


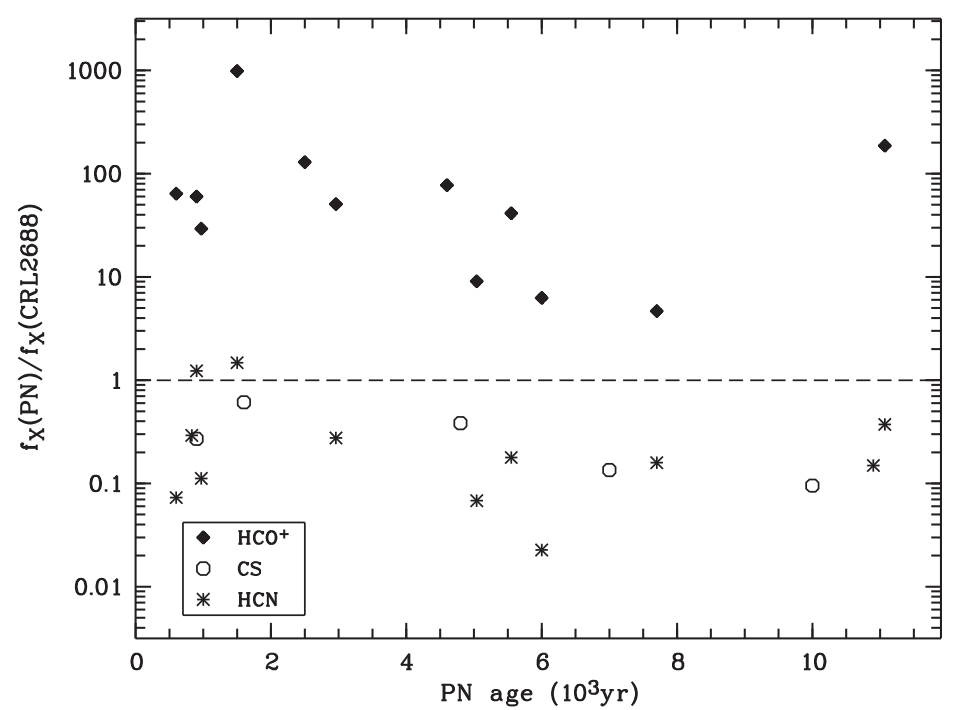

Figure 1. Molecular abundance (wrt $\mathrm{H}_{2}$ ) ratios between PNe and the PPN CRL 2688 versus PN ages. The data are taken from Bachiller et al. (1997), Zhang et al. (2008,2013), Edwards et al. (2014), and Schmidt \& Ziurys (2016). The dash line represents equivalent abundances.

$\mathrm{CH}_{2}+\stackrel{\text { e }}{\rightarrow} \mathrm{CH}$. The activation barrier for the formation of $\mathrm{CH}^{+}$may be overcome by the energy carried by shocks or vibrationally excited $\mathrm{H}_{2}$ in PDRs.

N-bearing molecules. CN has a relatively high abundance in $\mathrm{PNe}$ (Bachiller et al. 1997), which can be attributed to the photodissociation of HCN. Chemical models predict that, even considering the shielding of clumps, significant destruction of HCN in PNe occurs at a timescale of $\sim 10^{4} \mathrm{yrs}$ (Redman et al. 2003). However, this was not supported by recent observations that suggested an approximately constant $\mathrm{HCN}$ abundance during the PN evolution (Fig. 1, Schmidt \& Ziurys 2016). HNC, a less stable isomer of HCN, has generally increasing abundance in PNe with respect to PPNe (Bachiller et al. 1997). However, the $\mathrm{HNC} / \mathrm{HCN}$ isomer ratio in NGC 7027 appears abnormally low, which has not been well understood. $\mathrm{HC}_{3} \mathrm{~N}$ and $\mathrm{NH}_{3}$, unlike those in the AGB and PPNe, are rarely detected in $\mathrm{PNe} . \mathrm{HC}_{3} \mathrm{~N}$ was detected in $\mathrm{NGC} 7027$ with low abundance (Zhang et al. 2008). NGC 6302 shows $\mathrm{NH}_{3}$ emission (Bujarrabal et al. 2012). The two molecules can either be the remnant of circumstellar chemistry, or be reformed through neutral reactions in the dense, warm post-shock layers of PNe. The abundance of $\mathrm{N}_{2} \mathrm{H}^{+}$in NGC 7027 is extremely high (Josselin \& Bachiller 2003, Zhang et al. 2008). This might be due to the strong X-ray radiation which enhances the reaction $\mathrm{N}_{2} \stackrel{\mathrm{H}_{3}^{+}}{\longrightarrow} \mathrm{N}_{2} \mathrm{H}^{+}$. This molecule was detected in NGC6537 and NGC6302 as well (Edwards \& Ziurys 2013, Hebden 2014).

$S$ - and Si-bearing molecules. PNe are characterized with a lack of S- and Si-bearing molecules, presumably because of depletion onto dust grains. CS rotational lines are typically strong in C-rich AGB envelopes and PPNe, but never detected in C-rich PNe. Only a few O-rich PNe exhibit weak CS emission (Fig. 1, Edwards et al. 2014, Smith et al. 2016), probably suggesting that oxidation environments are favorable for sulfur in grains to be released into the gas phase. $\mathrm{HCS}^{+}$and $\mathrm{SiC}_{2}$ were only tentatively detected in NGC 7027 and NGC 6302, respectively (Zhang et al. 2008, Hebden 2014). The other uncommon molecules $\mathrm{SO}, \mathrm{SO}_{2}$, and $\mathrm{SiO}$ were detected in M2-28 (Edwards \& Ziurys 

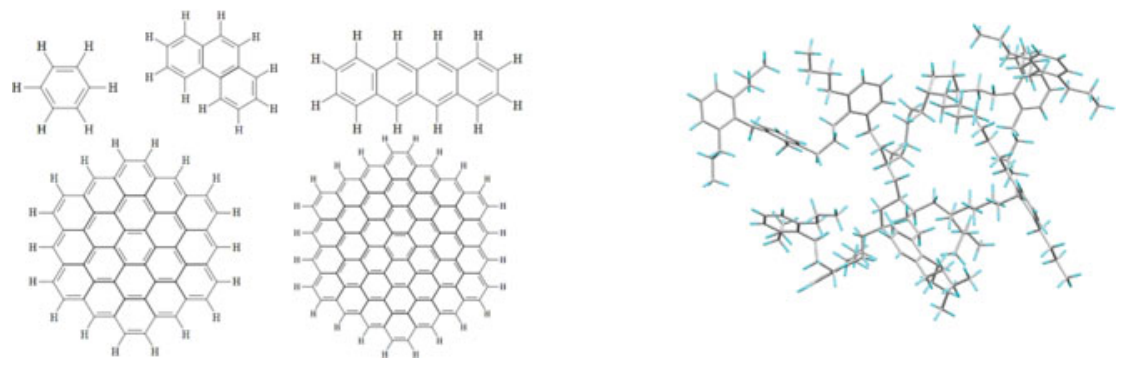

Figure 2. Schematic chemical structures of PAHs (left) and MAON (right).

2014). SiO, SO, and NS were revealed in the dense molecular regions of NGC 6302 by the SMA and ALMA observations (Hebden 2014, Santander-García et al. 2016).

$\mathrm{C}_{2} \mathrm{H}, \mathrm{c}-\mathrm{C}_{3} \mathrm{H}_{2}$, and $\mathrm{H}_{2} \mathrm{CO}$. The three compounds are present in young and evolved $\mathrm{PNe}$ (e.g. Woods \& Nyman 2005, Tenenbaum et al. 2009), suggesting that they can survive intense radiation fields. Initiating with acetylene, $\mathrm{C}_{2} \mathrm{H}$ and the cyclic molecule $c-\mathrm{C}_{3} \mathrm{H}_{2}$ can be formed through photochemistry. However, Fuente et al. (2003) found that the large $c-\mathrm{C}_{3} \mathrm{H}_{2}$ abundance in NGC 7027 is beyond the predictions of gas-phase chemistry models, and might arise from the photodestruction of large aromatic/aliphatic compounds. Although the formation of $\mathrm{H}_{2} \mathrm{CO}$ in PDRs might result from the radical reaction between $\mathrm{O}$ and $\mathrm{CH}_{3}$, it is unclear whether dust surface reactions $\left(\mathrm{CO} \stackrel{\mathrm{H}}{\longrightarrow} \mathrm{HCO} \stackrel{\mathrm{H}}{\rightarrow} \mathrm{H}_{2} \mathrm{CO}\right.$ ) followed by photodesorption also play a role.

\section{Complex organic species}

The presence of complex organics can be inferred from the unidentified infrared emission (UIE) bands that mainly manifest themselves as aromatic features at 3.3, 6.2, 7.7, 8.6, and $11.3 \mu \mathrm{m}$, aliphatic features at 3.4 , and $6.9 \mu \mathrm{m}$, and plateau emission around 8 , 12 , and $17 \mu \mathrm{m}$ (Tielens 2008, Kwok 2011, and reference therein). UIE bands have been observed in a variety of astronomical environments, and their fluxes show that the carrier might be the most abundant molecules in the ISM. Both low- and high-excitation PNe exhibit UIE bands, indicating that their carrier is a significant nebular component. A wide variety of materials has been proposed as potential carriers of the UIE bands, among which polycyclic aromatic hydrocarbon (PAH) is the most commonly accepted one (see Peeters 2013, for a recent review). However, the PAH hypothesis is not without problems (Kwok \& Zhang 2013, Zhang \& Kwok 2015). Kwok \& Zhang (2011) invoked an alternative model, mixed aromatic/aliphatic organic nanoparticles (MAONs), to interpret the UIE phenomenon. Fig. 2 shows the chemical structures of the PAHs and MAON. The spectral analysis shows that the aromatic/aliphatic component ratio increases during the transition from PPN to PN, suggesting that the aliphatic component may be processed to more stable aromatic rings by ultraviolet photons. The destruction of MAONs can provide a top-down route for the formation of smaller molecules. Nevertheless, no consensus has been reached concerning the UIE carrier. The MAON model was recently questioned (e.g. Li \& Draine 2012, Yang et al. 2016).

Recent observations of NGC 7027 provide support for the formation of the UIE carrier through grain-grain collisions in post-shock regions (Lau et al. 2016). UIE bands were also discovered in O-rich PNe. Infrared images of O-rich PNe show that UIE primarily originates from PDRs associated with the outer edge of central tori or dense knots, 

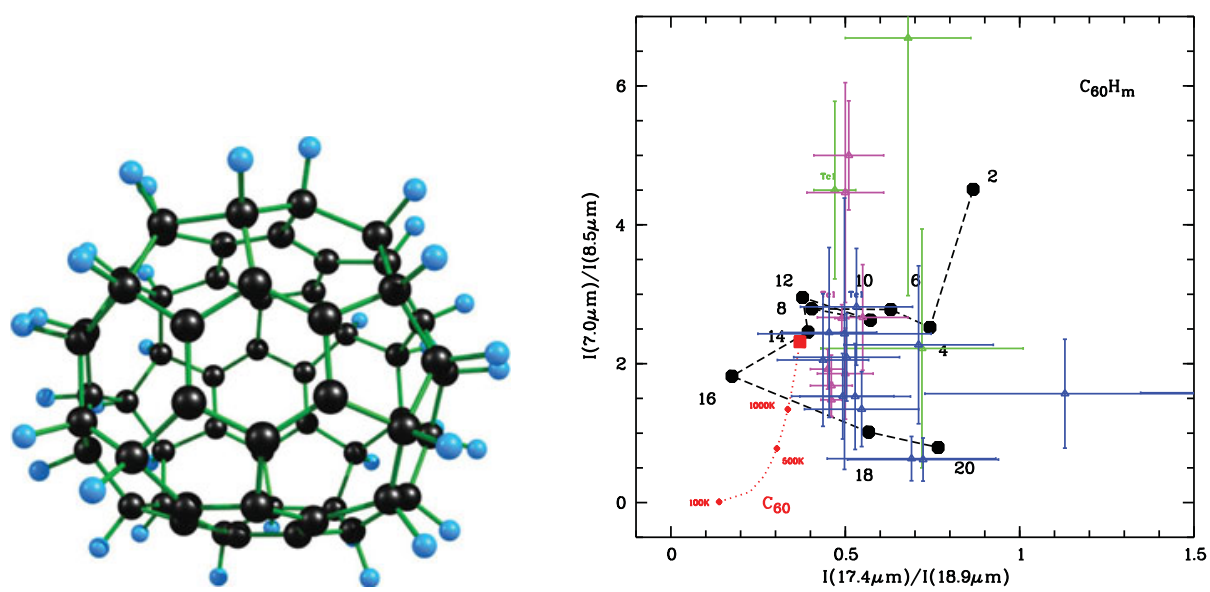

Figure 3. Left: Optimized structures of a $\mathrm{C}_{60} \mathrm{H}_{36}$ isomer. Right: Theoretical intrinsic strength ratios of the four $\mathrm{C}_{60} \mathrm{H}_{\mathrm{m}}$ backbone vibrations (filled circles connected by dashed line, with the $\mathrm{m}$ values marked). The triangles with error bars are the observed values taken from Bernard-Salas et al. (2012), García-Hernández et al. (2012), and Otsuka et al.(2014). The dotted curve represents the predicted ratios based upon the thermal excitation model of $\mathrm{C}_{60}$.

suggesting that the UIE carrier can be formed through a bottom-up process following the photodissociatoin of CO (Guzman-Ramirez et al. 2014, Cox et al. 2016).

\section{Fullerene-related compounds}

Given its high stability, $\mathrm{C}_{60}$, the best-known fullerene member, has been long believed to exist throughout circumstellar and interstellar environments (Kroto et al. 1985). Because of high symmetry, the vibrational modes of $\mathrm{C}_{60}$ are degenerated into four individual infrared features, making the astronomical search possible. Soon after the first detection in a young PN (Cami et al. 2010), $\mathrm{C}_{60}$ was discovered in various astronomical environments, suggesting that it could survive in very harsh conditions, though the formation route is under a controversial debate (see Cami et al. 2011 and this volume, Zhang \& Kwok 2013, Zhang et al. 2016, for the details). So far, 11 Galactic PNe and 11 Magellanic Cloud PNe have been detected with $\mathrm{C}_{60}$ (García-Hernández et al. 2012, Sloan et al. 2014, Otsuka et al. 2016). The detection rate is higher in more metal-poor environments. The presence of $\mathrm{C}_{60}$ in a PPN suggests that it can be formed within a timescale of $<10^{3} \mathrm{yrs}$ (Zhang \& Kwok 2011). Given its rather low ionization potential, $\mathrm{C}_{60}$ presumably exists largely in the cation form in the ISM. The notion of $\mathrm{C}_{60}^{+}$as one of the DIB carriers (Foing \& Ehrenfreund 1994) was recently verified by gas-phase experimental data, that is, five near-infrared DIBs were convincingly assigned to $\mathrm{C}_{60}^{+}$(Campbell et al. 2015,2016, Walker et al. 2015). A few DIBs were found to be enhanced in $\mathrm{C}_{60}$-containing nebulae (Iglesias-Groth \& Esposito 2013, Díaz-Luis et al. 2015), suggesting that they might have a circumstellar origin and probably be related to fullerenes or derivatives. Given their chemical activity and physical stability, fullerene-related compounds are good candidates for the DIB carrier (see Omont 2016, for a comprehensive discussion). Future studies should examine the spectra of fullerene compounds including hydrogenated fullerenes $\left(\mathrm{C}_{60} \mathrm{H}_{\mathrm{m}}\right), \mathrm{C}_{60}$ adducts, endofullerenes, hererofullerenes, buckyonions, and so on.

If mixed with $\mathrm{H}$ atoms, $\mathrm{C}_{60}$ can be readily hydrogenated into $\mathrm{C}_{60} \mathrm{H}_{36}$ (Fig. 3) in laboratory environments. Thus $\mathrm{C}_{60} \mathrm{H}_{\mathrm{m}}$ is highly likely to be present in $\mathrm{PNe}$, and may contribute to the formation of $\mathrm{H}_{2}$ by dehydrogenation (Cataldo \& Iglesias-Groth 2009). A tentative 
detection of $\mathrm{C}_{60} \mathrm{H}_{\mathrm{m}}$ in a $\mathrm{C}_{60}$-containing PPN has been presented by Zhang \& Kwok (2013). However, two $\mathrm{C}_{60}$-rich $\mathrm{PNe}$ do not exhibit any detectable $\mathrm{C}-\mathrm{H}$ feature (Díaz-Luis et al. 2016). Theoretical investigation indicated that with slight hydrogenation, the four $\mathrm{C}_{60}$-backbone vibration features at $7.0,8.5,17.4$, and $18.9 \mu \mathrm{m}$ are still visible but diminish their intensities in different degrees (Zhang et al. 2016). Therefore, $\mathrm{C}_{60}$-poor PNe might be more ideal targets for the search of $\mathrm{C}_{60} \mathrm{H}_{\mathrm{m}}$. The excitation mechanism of $\mathrm{C}_{60}$ bands is still unclear, hindering their use as a probe of PN environments (Brieva et al. 2016). As shown in Fig. 3, the observed intensity ratios are too dispersed to accord with the excitation model characterized with one single free parameter (i.e. fluorescence or thermal excitation models governed by the photon field energy and temperature, respectively), but can be well interpreted in terms of hydrogenation (Zhang et al., in preparation). This might provide circumstantial evidence for the presence of $\mathrm{C}_{60} \mathrm{H}_{\mathrm{m}}$ in $\mathrm{PNe}$.

\section{Conclusion}

A wealth of observations have demonstrated that molecules are an essential component in PNe. Even large molecules can be formed and/or survive during the PN evolution. The complex molecules blown from PNe into the ISM might contribute to the DIBs and seed the chemistry of molecular clouds. However, the complete picture of the chemistry in PNe is still far from clear. Systematic molecular line surveys of PNe are relatively scarce. Many molecules are discovered in only one or a few PNe. There is an absence of thorough understanding of the connection between the molecular composition and the properties of PNe. It is expected that new and important insights will be offered by the next generation instruments. Searching for fullerene-related compounds in PNe might provide crucial clues toward solving the DIB enigma. Further theoretical and experimental studies are badly needed.

\section{Acknowledgements}

I thank the SOC for inviting me to do this review talk. I am indebted to my colleagues who have closely collaborated with me in the study of circumstellar molecules, including Sun Kwok, Jun-ichi Nakashima, and Seyedabdolreza Sadjadi. Financial support for this work was provided by the Research Grants Council of the Hong Kong under grants HKU7073/11P and HKU7062/13P.

\section{References}

Aleman, I., Ueta, T., Ladjal, D., et al. 2014, A\& A, 566, A79

Bachiller, R., Forveille, T., Huggins, P. J., \& Cox, P. 1997, A\&AA, 324, 1123

Bell, T. A., Whyatt, W., Viti, S., \& Redman, M. P. 2007, MNRAS, 382, 1139

Bernard-Salas, J., Cami, J., Peeters, E., et al. 2012, ApJ, 757, 41

Black, J. H. 1978, ApJ, 222, 125

Brieva, A. C., Gredel, R., Jäger, C., Huisken, F., \& Henning, T. 2016, ApJ, 826, 122

Bujarrabal, V. 2006, in IAU Symp., 234, 193

Bujarrabal, V., Alcolea, J., Soria-Ruiz, R., et al. 2012, A\& A, 537, A8

Cami, J., Bernard-Salas, J., Peeters, E., \& Malek, S. E. 2010, Science, 329, 1180

Cami, J., Bernard-Salas, J., Peeters, E., \& Malek, S. E. 2011, in IAU Symp., 280, 216

Campbell, E. K., Holz, M., Gerlich, D., \& Maier, J. P. 2015, Nature, 523, 322

Campbell, E. K., Holz, M., Maier, J. P., et al. 2016, ApJ, 822, 17

Cataldo, F. \& Iglesias-Groth, S. 2009, MNRAS, 400, 291

Cernicharo, J., Liu, X.-W., González-Alfonso, E., et al. 1997, ApJL, 483, L65

Cernicharo, J., Agúndez, M., \& Guélin, M. 2011, in IAU Symp., 280, 237 
Cox, N. L. J., Pilleri, P., Berné, O., Cernicharo, J., \& Joblin, C. 2016, MNRAS, 456, L89

Díaz-Luis, J. J., García-Hernández, D. A., Kameswara Rao, N., et al. 2015, A\&6A, 573, A97

Díaz-Luis, J. J., García-Hernández, D. A., Manchado, A., \& Cataldo, F. 2016, A\&5A, 589, A5

Edwards, J. L. \& Ziurys, L. M. 2013, ApJL, 770, L5

Edwards, J. L., Cox, E. G., \& Ziurys, L. M. 2014, ApJ, 791, 79

Edwards, J. L. \& Ziurys, L. M. 2014, ApJL, 794, L27

Etxaluze, M., Cernicharo, J., Goicoechea, J. R., et al. 2014, A\& A, 566, A78

Foing, B. H. \& Ehrenfreund, P. 1994, Nature, 369, 296

Froebrich, D., Makin, S. V., Davis, C. J., et al. 2015, MNRAS, 454, 2586

Fuente, A., Rodriguez-Franco, A., Garcia-Burillo, S., et al. 2003, A\&A, 406, 899

García-Hernández, D. A., Manchado, A., García-Lario, P., et al. 2010, ApJL, 724, L39

García-Hernández, D. A., Villaver, E., García-Lario, P., et al. 2012, ApJ, 760, 107

Gómez, J. F., Rizzo, J. R., Suárez, O., et al. 2015a, A\&\&A, 578, A119

Gómez, J. F., Suárez, O., Bendjoya, P., et al. 2015b, ApJ, 799, 186

Guzman-Ramirez, L., Lagadec, E., Jones, D., et al. 2014, MNRAS, 441, 364

Hebden, K., 2014, Ph.D. Thesis

Huggins, P. J., Bachiller, R., Cox, P., \& Forveille, T. 1996, A\&̈A, 315, 284

Huggins, P. J., Bachiller, R., Planesas, P., Forveille, T., \& Cox, P. 2005, ApJS, 160, 272

Iglesias-Groth, S. \& Esposito, M. 2013, ApJL, 776, L2

Josselin, E. \& Bachiller, R. 2003, A\& A, 397, 659

Kimura, R. K., Gruenwald, R., \& Aleman, I. 2012, A\& A, 541, A112

Kroto, H. W., Heath, J. R., Obrien, S. C., Curl, R. F., \& Smalley, R. E. 1985, Nature, 318, 162

Kwok, S. 1994, PASP, 106, 344

Kwok, S. 2011, in IAU Symp., 280, 203

Kwok, S. \& Zhang, Y. 2011, Nature, 479, 80

Kwok, S. \& Zhang, Y. 2013, ApJ, 771, 5

Lau, R. M., Werner, M., Sahai, R., \& Ressler, M. E. 2016, ApJ, in press

Li, A. \& Draine, B. T. 2012, ApJL, 760, L35

Liu, X.-W., Barlow, M. J., Nguyen-Q-Rieu, et al. 1996, A $\& A$, 315, L257

Liu, X.-W., Barlow, M. J., Dalgarno, A., et al. 1997, MNRAS, 290, L71

Manchado, A., Stanghellini, L., Villaver, E., et al. 2015, ApJ, 808, 115

Marquez-Lugo, R. A., Ramos-Larios, G., Guerrero, M. A., et al. 2013, MNRAS, 429, 973

Millar, T. J. 2016, JPhCS, 728, 052001

Omont, A. 2016, A\& A, 590, A52

Otsuka, M., Kemper, F., Cami, J., Peeters, E., \& Bernard-Salas, J. 2014, MNRAS, 437, 2577

Otsuka, M., Kemper, F., Leal-Ferreira, M. L., et al. 2016, MNRAS, 462, 12

Pardo, J. R., Cernicharo, J., Goicoechea, J. R., Guélin, M., \& Ramos, A. A. 2007, ApJ, 661, 250

Park, J. A., Cho, S.-H., Lee, C. W., \& Yang, J. 2008, AJ, 136, 2350

Peeters, E. 2013, in IAU Symp., 297, 187

Qiao, H.-H., Walsh, A. J., Gómez, J. F., et al. 2016, ApJ, 817, 37

Redman, M. P., Viti, S., Cau, P., \& Williams, D. A. 2003, MNRAS, 345, 1291

Santander-García, M., Bujarrabal, V., \& Alcolea, J. 2012, A\&A, 545, A114

Santander-García, M., Bujarrabal, V., Alcolea, J., et al. 2016, $A \mathscr{E} A$, in press

Schmidt, D. R. \& Ziurys, L. M. 2016, ApJ, 817, 175

Sloan, G. C., Lagadec, E., Zijlstra, A. A., et al. 2014, ApJ, 791, 28

Smith, C. L., Zijlstra, A. A., \& Fuller, G. A. 2015, MNRAS, 454, 177

Tenenbaum, E. D., Milam, S. N., Woolf, N. J., \& Ziurys, L. M. 2009, ApJL, 704, L108

Tielens, A. G. G. M. 2008, ARAËA, 46, 289

Uscanga, L., Gómez, J. F., Suárez, O., \& Miranda, L. F. 2012, A $\& A$ A, 547, A40

van Hoof, P. A. M., van de Steene, G. C., Barlow, M. J., et al. 2010, A\& $A$, 518, L137

Walker, G. A. H., Bohlender, D. A., Maier, J. P., \& Campbell, E. K. 2015, ApJL, 812, L8

Wesson, R., Cernicharo, J., Barlow, M. J., et al. 2010, A\& $A$, 518, L144

Woods, P. M. \& Nyman, L.-Å. 2005, IAU Symp., 235, 326

Yang, X. J., Glaser, R., Li, A., \& Zhong, J. X. 2016, MNRAS, 462, 1551 
Zhang, Y. \& Kwok, S., Dinh-V-Trung 2008, ApJ, 678, 328-346

Zhang, Y. \& Kwok, S. 2011, ApJ, 730, 126

Zhang, Y. \& Kwok, S. 2013, EPESS, 65, 1069

Zhang, Y., Kwok, S., Nakashima, J.-i., \& Chau, W., Dinh-V-Trung 2013, ApJ, 773, 71

Zhang, Y. \& Kwok, S. 2015, ApJ, 798, 37

Zhang, Y., Kwok, S., \& Sadjadi, S. 2016, JPhCS, 728, 052004

Ziurys, L. M. 2008, IAU Symp., 251, 147

Zuckerman, B. \& Gatley, I. 1988, ApJ, 324, 501

\section{Discussion}

CHen: How do you prepare hydragenated fullerenes?

ZHANG: We did not prepare hydragenated fullerenes in experiments. These spectra were from theoretical calculations. We calculated the spectra of many isomers for each hydragenated C60, selected those with low energy, and combined them. The combined spectra can be used to compare with the observations. 